\title{
BRIDGING THE GAP OF ASSESSMENT AND FEEDBACK USING MARKUP - AN ANDROID APP FOR FAST MARKING AND FEEDBACK OF IN-CLASS ASSESSMENTS
}

\author{
Na Yao', Nickos Paltalidis', Luchan Wang ${ }^{2}$ \\ ${ }^{1}$ Queen Mary, University of London (UK) \\ ${ }^{2}$ Beijing University of Posts and Telecommunications (China)
}

\begin{abstract}
This paper introduces an innovative bespoke Android App used for in-class assessment in order to help lecturers to provide marks and feedback easily and efficiently. This work was inspired by Personal Development Programme (PDP), a module designed for the Joint Programme (JP) students between Queen Mary, University of London and Beijing University of Posts and Telecommunications [1]. The PDP is a compulsory module in the first three years of JP and includes a mix of skills and activities like presentation and public speaking, team working, and technical writing skills. In order to solve the issue of the delay from when the presentation is assessed to students getting individual feedback, a mobile app is developed to allow lecturer type comments and give marks during the assessment, and the saved comments and marks can be distributed to students without further delay.

The MarkUP app has been tested in PDP in-class assessment and improvement areas have been identified. Future work aims to fix application bugs and streamline information import and export between the app and the existing VLE system.
\end{abstract}

Keywords: Assessment, feedback, mobile app, mobile learning, higher education.

\section{INTRODUCTION}

In the last two decades, mobile learning in different forms have been adopted alongside traditional learning [2] [3]. Moreover, assessment as part of important aspect of the learning process has evolved too with electronic assessment [4]. With the development of powerful mobile devices and technologies, mobile app can be used in even more ways. This work is a first attempt in developing a Mobile app to assist lecturers during assessment in terms of recording comments and providing more timely feedback.

Personal Development Programme (PDP), is a module designed for the Joint Programme (JP) students between Queen Mary, University of London and Beijing University of Posts and Telecommunications [1]. The PDP is a compulsory module in the first three years of JP and includes a mix of skills and activities like presentation and public speaking, team working, and technical writing skills.

A typical PDP assessment involves a group presentation; particularly, in a semester there are up to 1200 students working on PDP tasks that involve in-class assessment of up to 240 group presentations. This large amount of assessments in a short time generates a problem, namely how a lecturer could efficiently mark the presentations and provide timely feedback to the students. Traditionally a lecturer assesses the in-class presentations by taking notes (either on paper or digitally), followed by giving an overall oral feedback to the whole class at the end of the assessment. In addition, after the assessment sessions, the lecturer has to transfer the written comments and mark for each presentation to a pre-defined marking sheet for each individual group, and the marking sheets are uploaded to a virtual learning environment (VLE) system, finally the marks and comments are distributed to students via the VLE system.

The main problem here is the mismatch between the format of notes taking (without a template) and the format of results distribution (pre-defined marking sheet) to students. Lecturer will have to transfer the notes and marks to the individual group marking sheet after assessment, and the sheer amount of groups makes this task very time consuming, thus generating a considerable delay for the students to get their individual group comments. Previous research has shown it is important to provide timely feedback [5] [6], and timeliness of feedback is listed as one of the key indicators in the Quality 
Assurance Agency's (QAA) guidelines on assessment and feedback, as outlined in their code of practice for assessing students [7]. In order to reduce the delay of providing feedback, over the last few years different methods have been trailed, such as involving more members of academic staff in the assessment. However this has not effectively solved the problem of marking and providing individual feedback as one lecturer still has to mark and give feedback to at least 10 different groups of students, and this method inevitably run the risk of increased marking discrepancy done by different people.

An innovative solution inspired by the increased adoption of Mobile apps in teaching and learning was proposed to solve this problem. The idea is to create an Android app as a final year project, and the aim of the app is to integrate the format of notes taking and marking while lecturer listening to presentations, then the comments and marks can be distributed to students afterwards without further delay.

\section{DESIGN AND IMPLEMENTATION}

\subsection{Requirements analysis}

\subsubsection{Functional Requirements}

Three interviews were carried out with the potential user, namely with the lecturer of PDP class, where requirements of the app were discussed and analysed. Each interview lasted 15 to 30 minutes, where developer and user identified the following fundamental requirements for PDP marking:

- Marking function. Lecturer can see a list of marking criteria (which is pre-defined in the app) and use text field to give marks, and type in comments. These marks and comments should also be saved in database once confirmed.

- Importing students groups function. Students are divided into groups and assessment is done in each individual group. Once group information (student members) is known, this can be imported into the app.

- Searching function. The lecturer should be able to search certain groups by entering group number, student number etc.

- Exporting marks and comments function. Once all the marking is done, the lecture is able to export the marks and comments into a spreadsheet, which can then be uploaded into existing VLE system for distribution.

- Editing existing and creating new marking criteria. PDP tasks can be in different format other than presentations. It is vital that this app provides the function of editing existing marking criteria, and the function of creating new marking criteria so new tasks or different marking criteria can be accommodated.

\subsubsection{Hardware Requirements}

In terms of hardware requirements, the app is designed to be used on Android tablet due to the ease of operation provided by larger screen size. Android programing is flexible because it does not need fixed hardware or software configurations. Android is open source and programmers can customize Android applications by access to the source on the Internet as they want. Android also provides supports to many features, such as a lightweight relational database (SQLite), connectivity with other devices, messaging etc, which is essential for the functionalities in this project.

\subsection{Design of interface and mapping of marksheet}

The user interface design of the project is driven by functions; flow chart in Figure 1 illustrates the functions. Designing user interface of the project follows the principles of simplicity and easy to use. Each screen reflects an activity. LinearLayout is used because there are several lists in the project. ScrollView enables markers to scan long content on the screen. Lists of members are settled by using TableLayout. 


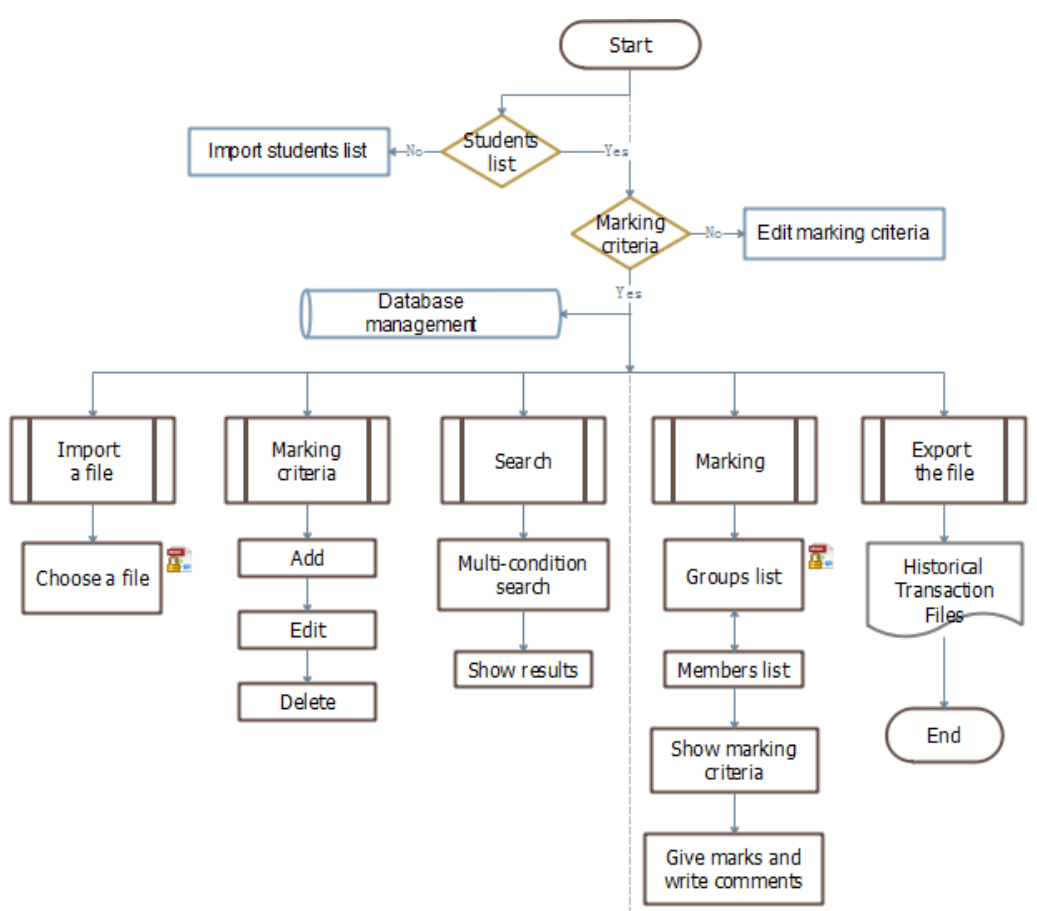

Figure 1. Function flow chart

One important task for the app is to transfer paper marksheet content into the app, especially the marking criteria. An example of the marksheet for the group presentation task is given below in Figure 2. The rows of the marksheet list different marking aspects and the columns indicates different grades.

\begin{tabular}{|l|l|l|l|l|l|}
\hline & Excellent & Very Good & Good & Satisfactory & Poor \\
\hline & $10-8$ & $7-6$ & $5-4$ & $3-2$ & $1-0$ \\
\hline Slides: & & & & & \\
\hline Format and font & & & & & \\
\hline Clear structure and coherent story & & & & & \\
\hline $\begin{array}{l}\text { Correct information in the slides (i.e. English } \\
\text { mistakes or factual mistakes) }\end{array}$ & & & & & \\
\hline Presentation: & & & & & \\
\hline Audible and clear & & & & & \\
\hline $\begin{array}{l}\text { Proper use of slides as a visual aid (i.e. not } \\
\text { reading from the slides) }\end{array}$ & & & & & \\
\hline $\begin{array}{l}\text { Speakers have 'presence' and adequate } \\
\text { confidence (i.e. appropriate eye contact) }\end{array}$ & & & & & \\
\hline Good interaction with audience & & & & & \\
\hline $\begin{array}{l}\text { Convey the message (presentation topic) } \\
\text { well }\end{array}$ & & & & & \\
\hline Answer the questions clear & & & & & \\
\hline Time management: stick to time (6-8 mins) & & & & & \\
\hline
\end{tabular}

Figure 2. Marksheet for group representation task

In order to make it clear on the screen, the column of paper marksheet is directly mapped on the screen, so that markers can easily see what specific area they are assessing. The marks are supposed to write down in the textbox following the criteria. However, the row of the table of papersheet is treated as a referenced standard, so they are placed below the marking descriptors in a different colour as a guideline for the lecturer.

\section{PRELIMENARY RESULTS}

The first version of the implemented app is illustrated here with screenshots of various functions.

The app has the initial function of importing different marking criteria for different assessment. Before the assessment, students and groups information can be imported into the app. 
During the assessment, the lecturer can select a certain group, type comments, enter marks for each criteria, and save all.

After assessment of all group work, lecturer can use the app to export the marks and feedback comments to a spreadsheet, and share them with students via email. With the aid of the app, all comments and marks are collected and saved in one go. This greatly reduces time consumption from duplicating paperwork on the lecturer side, and reduces delays of receiving feedback on the student side.

\subsection{Marking function}

Figure 3 and Figure 4 show the screenshots of the marking function. Users can see groups list and members list (from a spreadsheet that the user imported into the app), and they can fill the marksheet selecting marks based on predefined marking criteria, and typing comments in the text box for each group.

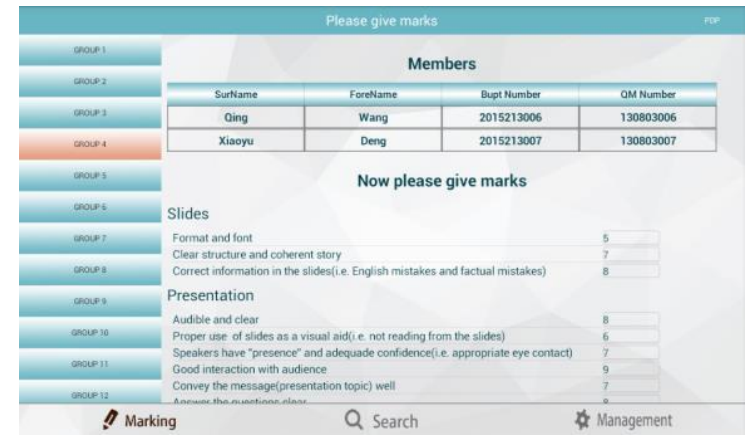

Figure 3 Marking function(1)

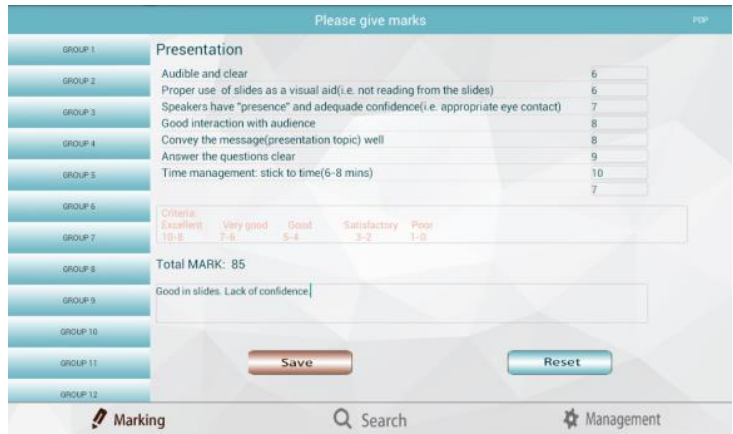

Figure 4 Marking function(2)

\subsection{Importing and exporting of spreadsheet}

The management of the app includes importing a new students list and exporting the students list with given marks.

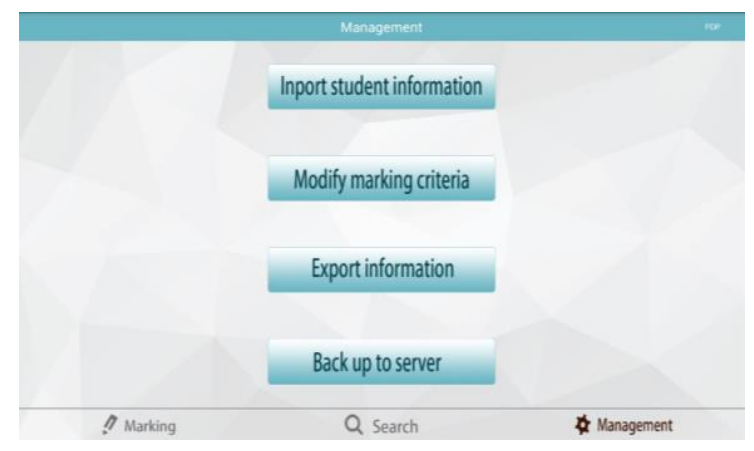

Figure 5 Management home page

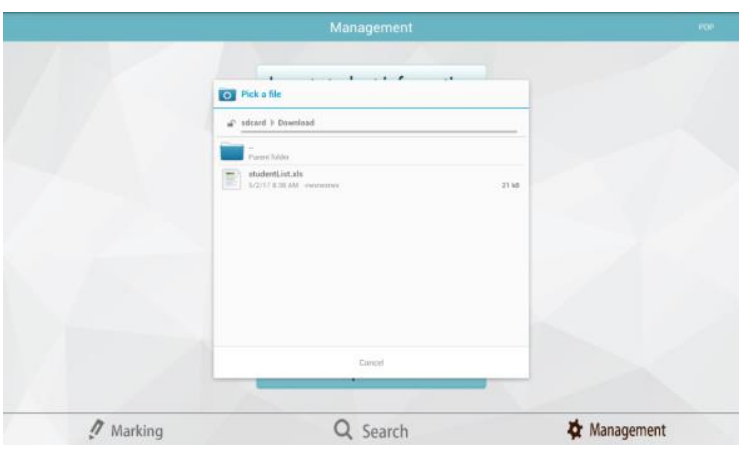

Figure 6 Importing file

\subsection{Editing and creating new marking criteria}

Considering that new PDP tasks with different marking criteria can be set in future years, the function for editing and creating new marking criteria is important. Figure 7 illustrates the function of creating new marking criteria, where the tasks and items of marking can be added and deleted, together with the total mark for each item. 


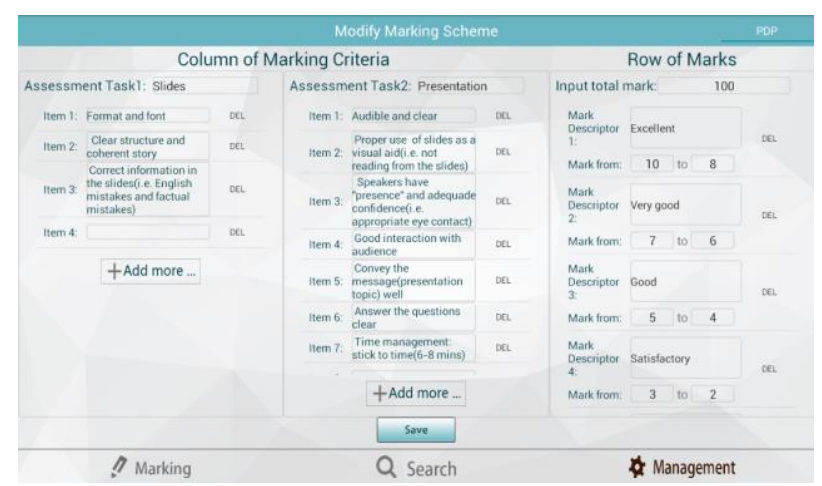

Figure 7 Editing and creating new marking criteria

\subsection{Search function}

Given the large number of groups and students, it will be useful to have a search function so lecturer can locate the correct group or students quickly.

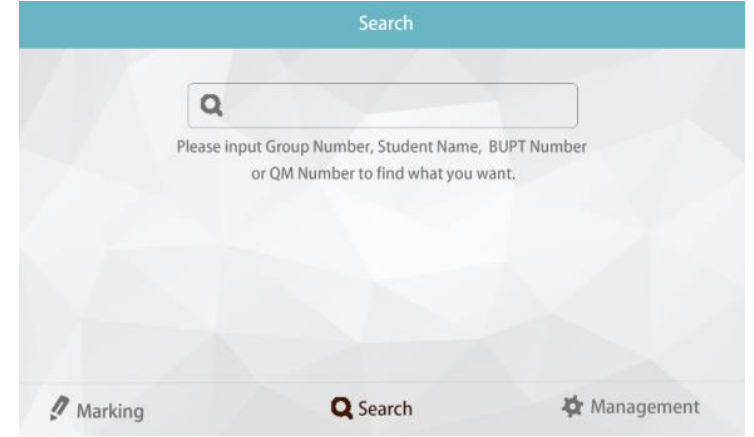

Figure 8 Search page

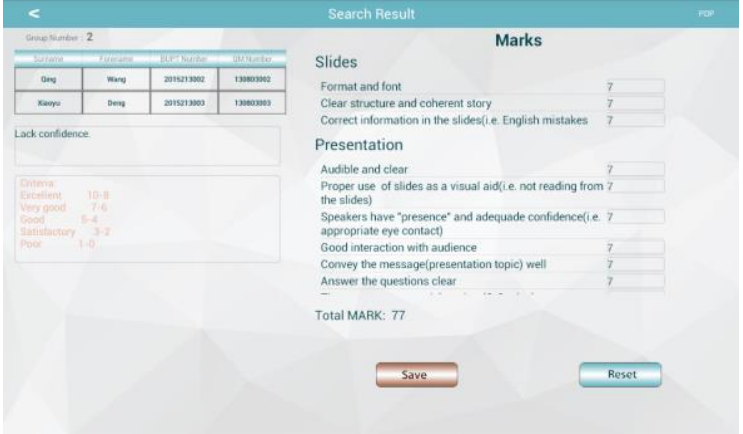

Figure 9 Search results

\subsection{Test and results}

The implemented app was tested by the lecturer of first year PDP class during the marking of an inclass assessment of 120 group presentations. Presentations were organised within a week of total 20 hours. Testing aimed to evaluate four main aspects of the app, namely if the app a) meets the requirements that guided its design and development, b) responds correctly to all kinds of inputs, c) performs its functions within an acceptable time, d) is sufficiently usable.

The initial results has been positive; using the proposed app, user (lecturer) successfully a) imported the groups/students details, b) pre-defined the marking criteria, c) entered marks and comments for each group, d) exported a spreadsheet with the marks and comments. The testing proved that the app provides an effective and accurate way to record marks and comments, especially for in-class assessments where real time evaluation is required and the time for marking is restricted. Particularly, user notes that the marks recoding activity was reduced by 3 minutes for each group, and marks and comments were distributed to students a couple of hours after the completion of the assessments, where traditionally written feedback were given 10 to 15 days after the presentation day.

It is also noted that improvements are required in order to improve the usability, the aesthetics, and the easy navigation across the app, particularly for the marking function:

- First, convert the "mark" text fields into drop down lists so the lecturer can easily pick a mark from the predefined marking scale;

- an auto-save function after marks or comments are entered will further benefit the lecturer, as the lecturer don't need to worry about clicking the "save" button.

- an auto-navigation to the next group's "marksheet" after marks or comments are entered/saved. 


\section{FUTURE WORK}

In addition, user has identified some further requirements where authors plan to develop in future, giving emphasis on the existing functions of the app as well as on a new function:

\section{Existing functions}

- Marking function: editing and creating new marking criteria can be re-modelled as the format of an advanced scoring rubric, so the lecturer can create different criteria freely; particularly lecturer will have the opportunity to set the evaluative criteria, give quality definitions for the marking descriptors at different levels of performance, and a scoring strategy to rate each criteria.

- Exporting marks and comments function: Convert filled scoring rubric into a feedback report and distribute it to each group by the existing VLE system and/or by email; namely a detailed feedback about the quality of the work will be available for the students immediately after the completion of the assessments, providing a basis for direct self-evaluation and reflection.

Additional function

- Performance Analysis function: Provide an analysis of students' assessment performance in a dashboard format; namely to analyse students 'grades from multiple perspectives (e.g. max, min, avg grade) and to demonstrate into different graphical representations.

\section{CONCLUSIONS}

This paper addresses a particular issue of providing timely feedback to module for the Joint Programme students where large groups of students are involved, and presents an innovative solution using mobile app. After following the process of requirement analysis, design and implementation, a first version of the app is developed and tested. Initial test has shown a substantial improvement to the time consumed by lecturer on notes taking and marking, and allowing students to get their feedback faster than previous method. Further areas to improve the app have also been identified.

\section{REFERENCES}

[1] L. Cuthbert, N. Yao, B. Chib, Y. Ying and Y. Wang, "A Success Story in Inter-Disciplinary, InterContinental Engineering Education" in Proceedings of IEEE conference of Transforming Engineering Education: Creating Interdisciplinary Skills for Complex Global Environments, Dublin, Ireland, April 2010.

[2] J. Traxler, "Defining Mobile Learning," in Proceedings of IADIS International Conference on Mobile Learning, pp. 261-266, 2005.

[3] A. Kukulska-Hulme, M. Sharples, M. Milrad, I. Arnedillo-Sánchez, and G. Vavoula, "The Genesis and Development of Mobile Learning in Europe," in Combining E-Learning and MLearning: New Applications of Blended Educational Resources (D. Parsons eds.),pp.151-177, Hershey, PA: Information Science Reference (an imprint of IGI Global), 2011.

[4] R. Brink and G. Lautenbach, "Electronic assessment in higher education," Educational Studies, vol. 37, no. 5, pp. 503-512. 2011.

[5] D. Nicol, "From Monologue to Dialogue: Improving Written Feedback Processes in Mass Higher Education," Assessment \& Evaluation in Higher Education, vol 35, no. 5, pp. 501-517, 2010.

[6] C. Vrasidas and M. S. Mcisaac, "Factors Influencing Interaction in an Online Course," American Journal of Distance Education, vol. 13, no. 3, pp. 22-36, 1999.

[7] Quality Assurance Agency, "UK Quality Code for Higher Education - Chapter B6: Assessment of students and the recognition of prior learning, " 2013, retrieved from http://www.qaa.ac.uk/publications/information-and-guidance/uk-quality-code-for-highereducation-chapter-b6-assessment-of-students-and-the-recognition-of-prior-learning1 